\section{Effectiveness of ultrasonography and shear wave sonoelastography in Sjögren syndrome with salivary gland involvement}

\author{
Yunus Emre Oruk ${ }^{1,2}$, Mehmet Burak Çildağ ${ }^{2}$, Can Zafer Karaman², Songül Çildağ ${ }^{3}$ \\ 'Department of Diagnostic Radiology, Muğla Menteșe State Hospital, Muğla, Departments \\ of ${ }^{2}$ Diagnostic and Interventional Radiology and ${ }^{3}$ Immunology and Allergy, Adnan Menderes \\ University, Aydin, Turkey
}

Purpose: This study investigated the effectiveness of major salivary gland ultrasonography (MSGUS) using a new grading system and shear wave elastography (SWE) in evaluating the major salivary glands of patients with Sjögren syndrome (SjS).

Methods: This prospectively-designed cross-sectional study included 49 SjS patients and 49 healthy controls. Major salivary glands were examined with gray-scale ultrasonography and SWE. A new grading system for salivary glands was developed using MSGUS findings. The sensitivity, specificity, positive predictive value (PPV), and negative predictive value (NPV) of MSGUS and shear wave velocity (SWV) values were investigated. The MSGUS grading system and SWV values were evaluated together by logistic regression analysis. A cutoff value of SWE for salivary glands was determined.

Results: The sensitivity, specificity, PPV, and NPV of MSGUS were $69.4 \%, 73.5 \%, 72.3 \%$, and $70.6 \%$ for the submandibular gland and $69.4 \%, 65.3 \%, 66.7 \%$, and $68.1 \%$ for the parotid gland, respectively. The mean SWV values of the parotid and submandibular glands were significantly higher in SjS patients than in controls $(P<0.05)$. The sensitivity, specificity, PPV, and NPV for the submandibular gland (cutoff, $1.95 \mathrm{~m} / \mathrm{s}$ ) and the parotid gland (cutoff, $2.39 \mathrm{~m} / \mathrm{s}$ ) were $69.4 \%, 52 \%, 59.1 \%$, and $63 \%$ and $82.7 \%, 83.7 \%, 83.5 \%$, and $82.8 \%$, respectively. Adding SWE to the parotid gland grading system increased the sensitivity and specificity (sensitivity, 82.7\%; specificity, 83.7\%).

Conclusion: Evaluating the salivary glands using MSGUS with a new grading system and SWE may contribute to the diagnosis of SjS. The combination of MSGUS and SWE may be a promising tool for diagnosing SjS.

Keywords: Parotid gland; Elastography; Submandibular gland; Ultrasonography

\section{Introduction}

Sjögren syndrome (SjS) is a chronic, inflammatory, autoimmune disease characterized by xerostomia and xerophthalmia, the main histopathological feature of which is lymphocytic infiltration, affecting exocrine glands such as salivary and lacrimal glands [1]. In addition to the clinical and laboratory data

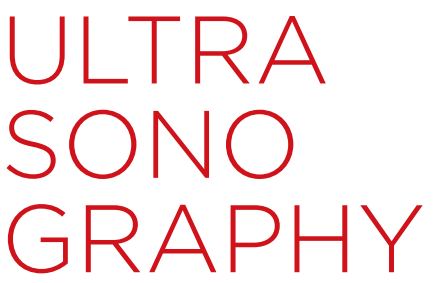

\section{ORIGINAL ARTICLE}

https://doi.org/10.14366/usg.21014 pISSN: 2288-5919 • elSSN: 2288-5943 Ultrasonography 2021;40:584-593

Received: January 19, 2021

Revised: March 20, 2021

Accepted: March 23, 2021

Correspondence to:

Yunus Emre Oruk, MD, Department of Diagnostic Radiology, Muğla Menteșe State Hospital, Muğla 48000, Turkey

Tel. $+90-534-7173915$

Fax. +90-252-213-10-13

E-mail: dr.yunusemreoruk@hotmail. com

This is an Open Access article distributed under the terms of the Creative Commons Attribution NonCommercial License (http://creativecommons.org/ licenses/by-nc/4.0/) which permits unrestricted noncommercial use, distribution, and reproduction in any medium, provided the original work is properly cited.

Copyright @ 2021 Korean Society of Ultrasound in Medicine (KSUM)

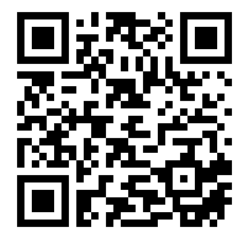

How to cite this article:

Oruk YE, Çildağ MB, Karaman CZ, Çildağ S. Effectiveness of ultrasonography and shear wave sonoelastography in Sjögren syndrome with salivary gland involvement. Ultrasonography. 2021 Oct;40(4):584-593. 
used to determine salivary gland involvement in the 2002 AmericanEuropean Consensus Group (AECG) criteria for the diagnosis of SjS, findings from salivary gland scintigraphy, labial salivary gland biopsy, non-stimulated sialometry, and sialography have been accepted as diagnostic criteria [2-4]. However, imaging methods were excluded from the diagnostic criteria in the most recent scoring-based criteria [5]. Sialography and labial salivary gland biopsy are important but invasive methods for the diagnosis of SjS. Scintigraphy is a sensitive but nonspecific method, and these methods may cause falsepositive results [2,3]. In recent studies, it has been reported that major salivary gland ultrasonography (MSGUS) is an inexpensive, easily accessible, radiation-free, reproducible method that has high accuracy in the diagnosis of SjS [2,3]. In addition, MSGUS has been proposed as a convenient and noninvasive alternative to sialography, sialoscintigraphy, and labial salivary gland biopsy for the diagnosis and classification of SjS [6-8]. Studies that scored major salivary gland parenchymal changes in SjS according to grayscale ultrasonography (US) findings in the literature have reported a wide range of sensitivity $(43 \%-90 \%)$ and specificity $(84 \%$ $100 \%)[2-4,7,9]$. More recently, a simplified scoring system based on parenchymal heterogeneity in gray-scale US examinations, which is relatively easy to use, has been created $[10,11]$. However, the wide variety in diagnostic performance may result from the use of different semi-quantitative and operator-dependent scoring systems for parenchymal changes. Shear wave elastography (SWE) is an evolving US imaging modality in which shear waves are generated by a specially produced transducer used to differentiate benign versus malignant lesions and to obtain quantitative measurements of tissue stiffness in many organs and tissues [12-14]. Typically, shear waves are generated by the US radiation force in tissue with an acoustic repulsion pulse, and the shear wave propagation velocity is measured. The measurement is used alone or converted into the Young modulus for a quantitative assessment of tissue stiffness [15]. Several recent studies have shown that SWE of the major salivary glands is a potentially useful parameter for the diagnosis of primary SjS [16-18]. However, no studies have compared the diagnostic performance of MSGUS scores and shear wave velocity (SWV) values for the diagnosis and classification of SjS. The aim of this study was to compare the diagnostic performance of MSGUS using a new grading system and SWE imaging for SjS with major salivary gland involvement, and to evaluate the diagnostic contribution of SWE to MSGUS.

\section{Materials and Methods}

\section{Compliance with Ethical Standards}

This prospectively-designed cross-sectional study was approved by the Institutional Review Board of Noninvasive Clinical Studies at the Muğla Sıtkı Kocman University Faculty of Medicine (Protocol number: 72855364-050.01.04.00.81/17625). Written informed consent forms in accordance with the Helsinki Declaration were obtained from all participants.

\section{Study Group}

Between September 2018 and January 2020, 49 SjS patients (including primary and secondary SjS), many of whom were newly diagnosed, were included in this study. The diagnosis of SjS was made according to the AECG criteria, which included a standard clinical examination performed by an experienced rheumatologist, serological and laboratory tests, ocular tests, and salivary gland biopsy (for 35 patients). Forty-nine healthy adult volunteers without rheumatologic or salivary gland diseases were recruited as the control group, and sonographic examinations (MSGUS and SWE) were performed in these volunteers. The exclusion criteria were an inability to assume the appropriate position for the US procedure, the use of parasympatholytic drugs or drugs affecting the salivary glands, the diagnosis of sarcoidosis, hepatitis $C$ and human immunodeficiency virus infection, and a history of radiotherapy in the head and neck region. Whether the patients were diagnosed with SjS was known prior to imaging. The focus score results (graded between 0 and 4 according to mononuclear cell infiltration) and the duration of disease were recorded in the SjS patients.

\section{Imaging Technique: MSGUS Grading}

In this study, Samsung Medison RS80A Prestige ultrasound system (Samsung Medison Co. Ltd., Seoul, Korea) equipped with a linear 3-12 MHz probe was used in MSGUS. During the scan, participants were in a supine position and the ultrasound probe was gently coupled to the body surface with a sufficient amount of ultrasound gel. The bilateral parotid glands and submandibular glands were evaluated. In MSGUS, the following parameters were evaluated in the longitudinal and transverse planes: echogenicity, margin, border features, parenchymal homogeneity, and internal structure (hypoechoic areas, cysts, echogenic bands/reflections, calcifications, and ductal dilatations). A new grading system was developed using the findings of gray-scale US in light of the current literature $[2,3,7]$. This new grading system takes a value between 0 and 10, based on the sum of four scoring parameters. The median value for the submandibular and parotid glands of all cases was used as the cutoff value. According to the cutoff value, a score of $0-1$ points was classified as grade 0 (no involvement), a score of 2-4 points as grade 1 (mild involvement), a score of 5-7 points as grade 2 (moderate involvement), and a score of 8-10 points as grade 3 (severe involvement) (Figs. 1-3). All ultrasound acquisitions and 
grading were performed by the same physician with 5 years of grayscale US experience.

\section{Imaging Technique: SWE Imaging}

SWE was performed with a linear transducer with a frequency range of 2-9 MHz, using an elastography application working with the acoustic radiation force imaging-based point SWE (pSWE) technique. The bilateral parotid glands and submandibular glands were identified under the guidance of gray-scale imaging. After the transducer was placed perpendicular to the measurement area, at least 10 SWE measurements were obtained in the different regions of the salivary gland (e.g., central, peripheral, and subcapsular areas) without visible vessels of each salivary gland and cystic areas during breath-holding. Measurements were made with a region of interest measuring $0.5 \mathrm{~cm} \times 0.5 \mathrm{~cm}$ with a depth range of $0.5-3$ $\mathrm{cm}$. Each SWE measurement gave an estimate of SWV in meters per second and kilopascals. For each salivary gland measurement, an attempt was made to keep the interquartile range-to-median ratio (IQR/M) below $30 \%$ and the reliability measurement index (RMI) value at 0.4 and above [19-22] (Figs. 4, 5). All SWE measurements were performed by the same physician with 3 years of SWE imaging

\section{Parenchyma echogenicity scoring \\ 0 : Normal echogenic signal \\ 1: Decreased echogenic signal}

\section{Parenchyma homogeneity scoring}

0 : Homogeneous parenchyma structure

1: Minimal heterogeneous parenchyma structure

2: Prominent heterogeneous parenchyma structure

3: Gross-coarse heterogeneous parenchyma structure

Internal structure scoring

0: Hypoechoic area/no echogenic band-reflection

1: Several diffuse hypoechoic areas/echogenic bandreflection

2: Multiple scattered hypoechoic areas/echogenic bandreflection and multiple cysts

3: Numerous scattered hypoechoic areas/echogenic bandreflection and multiple cysts or calcifications

Edge/Border (contour) scoring

0 : Smooth boundary properties

1: Partially identifiable, focal irregular border features

2: Fuzzy boundary features whose boundaries cannot be clearly defined

3: Boundary features that cannot be identified, cannot be distinguished from the surrounding parenchyma

0-1 points: grade 0 (no involvement)

2-4 points: grade 1 (mild involvement)

5-7 points: grade 2 (moderate involvement)

8-10 points: grade 3 (severe involvement)

Fig. 1. Major salivary gland ultrasonography points and grading method of the submandibular and parotid glands. experience. Intraobserver and interobserver agreement was not evaluated.

The diagnostic performance of MSGUS with the new grading system and SWV values in distinguishing salivary gland involvement and the diagnostic contribution of SWE to gray-scale US were evaluated. The relationships of the duration of the disease and focus scores with the mean SWV values in SjS patients were investigated.

\section{Statistical Analysis}

The data were statistically analyzed using SPSS version 22 (IBM Corp., Armonk, NY, USA). The conformity of numerical data to a normal distribution was evaluated by the Kolmogorov-Smirnov test and the skewness-kurtosis test, and hypothesis tests were determined according to the availability of parametric assumptions. Categorical data are shown as number and percentage, while quantitative data are shown as median (range) and mean and standard deviation. Statistical significance was investigated using the Mann-Whitney $U$ and Kruskal-Wallis tests for continuous variables. Categorical variables were evaluated using the Spearman square or Fisher exact chi-square test. The cutoff values were determined from a receiver operating characteristic (ROC) curve and the optimal cutoff point was identified according to the Youden test. The sensitivity, specificity, and area under the ROC curve (AUROC) were used as diagnostic performance indicators. The diagnostic performance obtained by using MSGUS and SWE together for the diagnosis of SjS was also evaluated by multiple binary logistic regression. The relationships of the parameters and their odds ratios (ORs) were investigated. A P-value of less than 0.05 , reflecting a $95 \%$ level of confidence, was considered to indicate statistical significance.

\section{Results}

This study included a total of 98 participants ( 11 men and 87 women). The SjS group included 49 patients ( 7 men and 42 women) with a mean age of $51.2 \pm 13.4$ years (range, 18 to 78 years). The control group included 49 healthy volunteers ( 4 men and 45 women) with a mean age of $47.4 \pm 12.5$ years (range, 21 to 78 years). There was no statistically significant difference between the patient and control groups in terms of age and sex $(P>0.05)$. The mean disease duration in the SjS group was $3.53 \pm 3.00$ years, and the percentage of newly diagnosed patients was $36.7 \%(n=18)$. Patients with a disease duration of $0-5$ years constituted $73.5 \%$ $(n=36)$ of all patients. The symptoms of all newly diagnosed patients (especially dry mouth and dry eyes) started at least 3 months before, and at most 6 months before, the US examinations. Labial salivary gland biopsy was performed in $71.4 \%(n=35)$ of the patients. 


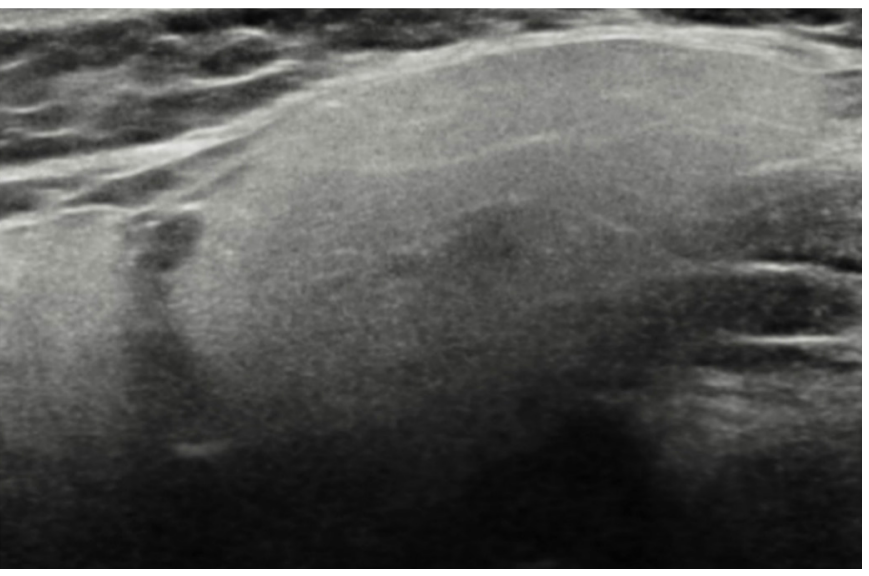

A

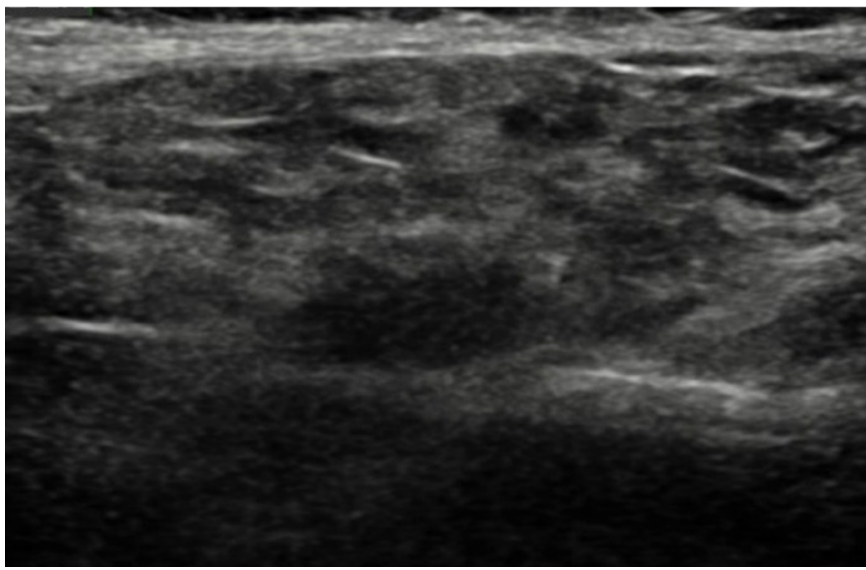

C

Fig. 2. MSGUS grading for the submandibular gland.

A. Normal submandibular gland is described as grade 0. B. Submandibular gland with decreased echogenic signal, focal irregular border features minimally heterogeneous parenchyma structure, several hypoechoic areas and echogenic bands are described as grade 1. C. Submandibular gland with decreased echogenic signal, uncertain boundaries, prominent heterogeneous parenchyma structure, and multiple scattered hypoechoic areas and echogenic bands are described as grade 2. D. Submandibular gland with decreased echogenic signal, grosscoarse heterogeneous parenchyma structure, unidentified boundaries, numerous scattered hypoechoic areas and echogenic bands are described as grade 3. MSGUS, major salivary gland ultrasonography.

The focus score was 0 in $18.4 \%$ of patients $(n=9), 1$ in $16.3 \%$ of patients ( $n=8), 2$ in $8.2 \%$ of patients ( $n=4), 3$ in $20.4 \%$ of patients $(n=10)$, and 4 in $8.2 \%$ of patients $(n=4)$.

In the SjS patient group, the distribution of grades for the bilateral submandibular glands was as follows: grade $0,28.6 \%(n=14)$; grade $1,34.7 \%(n=17) ;$ grade $2,16.3 \%(n=8)$; and grade $3,20.4 \%$ $(n=10)$. The mean MSGUS grade of the submandibular glands was $1.29 \pm 1.1$. In the control group, the distribution of grades was as follows: grade $0,73.5 \%(n=36)$ and grade $1,26.5 \%(n=13)$. The mean MSGUS grade of the submandibular glands was $0.27 \pm 0.45$. In the evaluation of the bilateral parotid glands, the distribution of grades in the patient group was as follows: grade $0,28.6 \%(n=14)$; grade $1,32.7 \%(n=16) ;$ grade $2,22.4 \%(n=11) ;$ and grade 3 ,

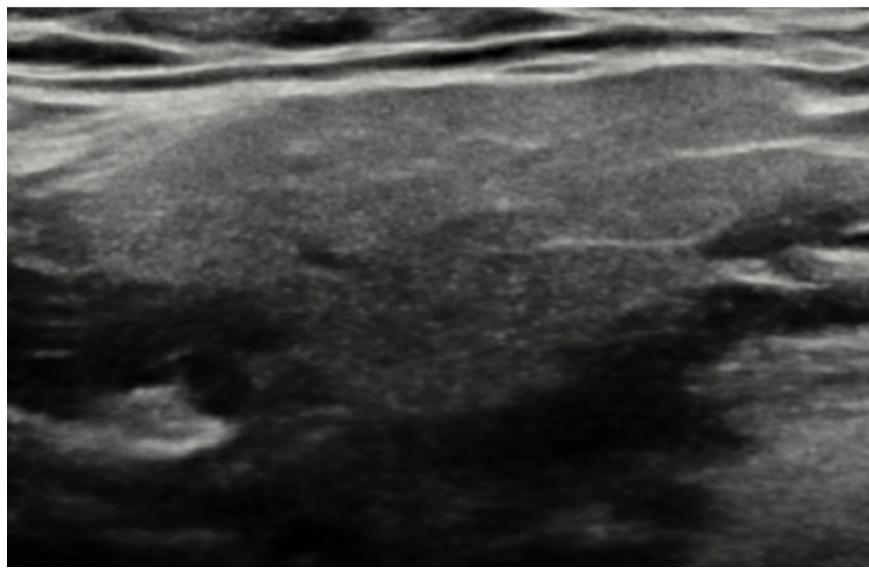

B

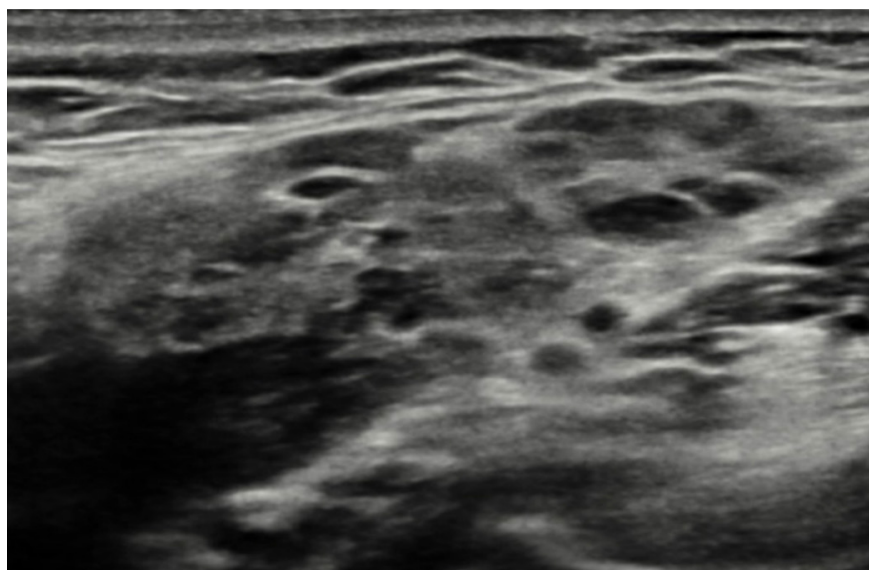

D 


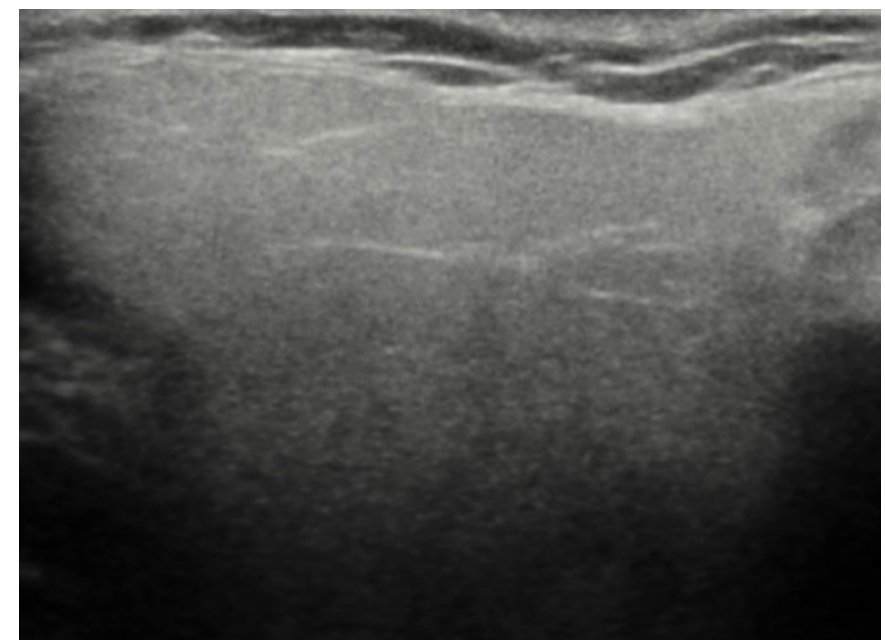

A

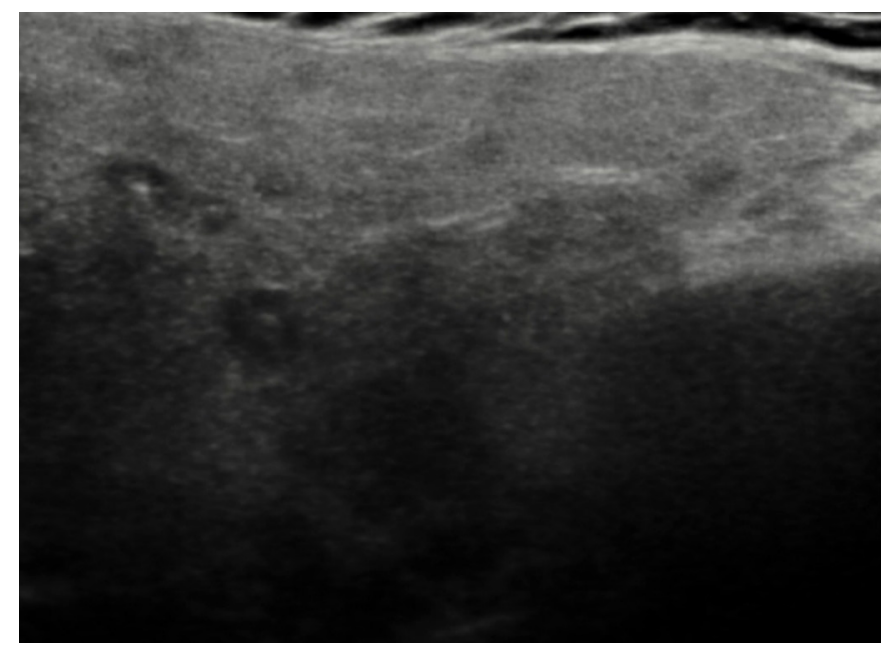

C

Fig. 3. MSGUS grading for the parotid gland.

A. Normal parotid gland is described as grade 0. B. Parotid gland with decreased echogenic signal, focal irregular border features, minimally heterogeneous parenchyma structure, several hypoechoic areas and echogenic bands are described as grade 1. C. Parotid gland with decreased echogenic signal, uncertain boundaries, prominent heterogeneous parenchyma structure, and multiple scattered hypoechoic areas and echogenic bands are described as grade 2. D. Parotid gland with decreased echogenic signal, gross-coarse heterogeneous parenchyma structure, unidentified boundaries, numerous scattered hypoechoic areas and echogenic bands are described as grade 3. MSGUS, major salivary gland ultrasonography.

Table 1. MSGUS grading of the submandibular and parotid glands

\begin{tabular}{lccccccc}
\hline & \multicolumn{4}{c}{ Group 1 $(\mathrm{n}=49)$} & & \multicolumn{2}{c}{ Group 2 $(\mathrm{n}=49)$} \\
\cline { 2 - 4 } \cline { 7 - 8 } & 0 & 1 & 2 & 3 & & 0 & 1 \\
\hline SMG & $14(28.6)$ & $17(34.7)$ & $8(16.3)$ & $10(20.4)$ & & $36(73.5)$ & $13(26.5)$ \\
PG & $14(28.6)$ & $16(32.7)$ & $11(22.4)$ & $8(16.3)$ & & $32(65.3)$ & $17(34.7)$ \\
\hline
\end{tabular}

Values are presented as number (\%).

MSGUS, major salivary gland ultrasonography; group 1, patient group; group 2, control group; SMG, submandibular gland; PG, parotid gland.

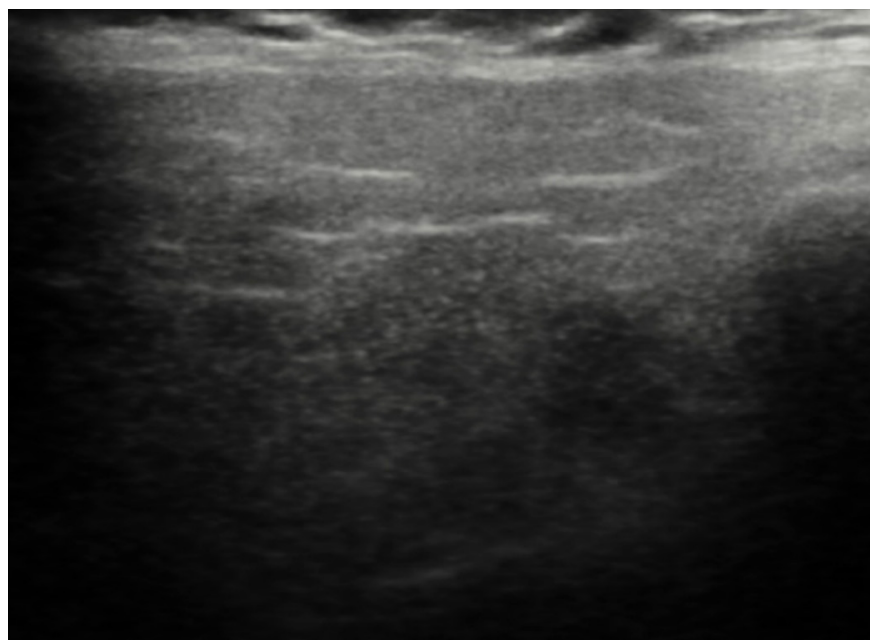

B

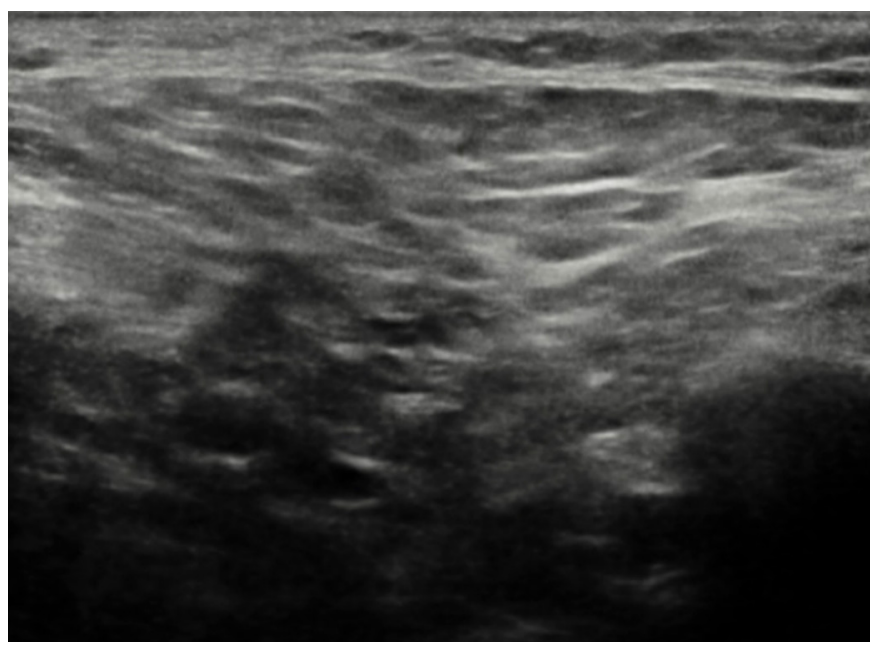

D 


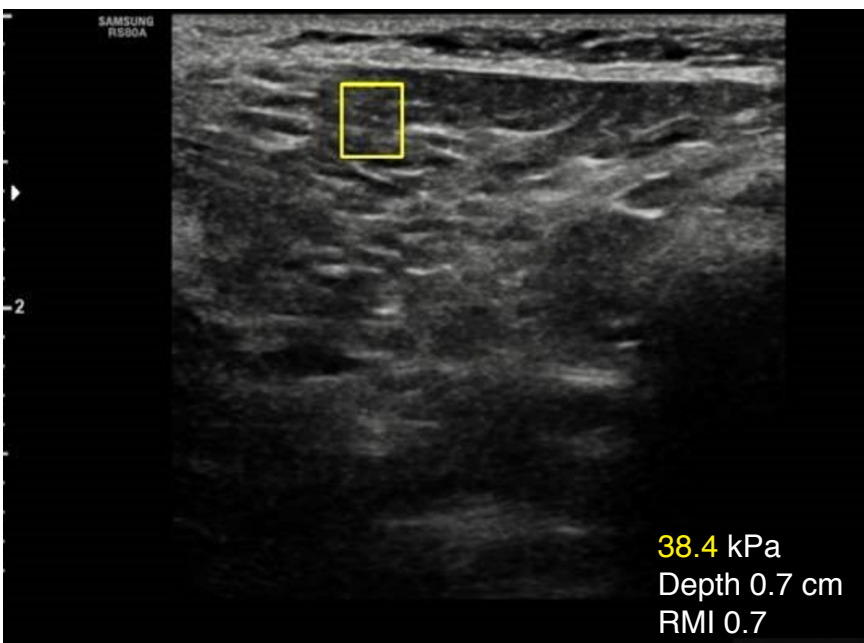

S.Shearwave Stiffness $(\mathrm{kPa}) \quad$ Stiffness $(\mathrm{m} / \mathrm{s}) \quad$ Depth $(\mathrm{cm}) \quad \mathrm{RMI}$

\begin{tabular}{crrrr}
\hline 1 & 23.7 & 2.81 & 0.7 & 0.8 \\
2 & 34.1 & 3.37 & 0.7 & 0.6 \\
3 & 36.5 & 3.49 & 0.7 & 0.7 \\
4 & 31.2 & 3.22 & 0.7 & 0.7 \\
5 & 23.3 & 2.79 & 0.7 & 0.8 \\
6 & 33.3 & 3.33 & 0.7 & 0.6 \\
7 & 36.8 & 3.50 & 0.7 & 0.8 \\
8 & 36.8 & 3.50 & 0.7 & 0.7 \\
9 & 36.6 & 3.49 & 0.7 & 0.8 \\
10 & 38.4 & 3.58 & 0.7 & 0.7 \\
Median & 35.3 & 3.43 & & \\
IQR/M & 21.2 & 11.15 & & \\
\hline
\end{tabular}

Fig. 4. Shear wave elastography (SWE) measurements in the parotid gland. The figure presents SWE measurements of the parotid gland. For each salivary gland measurement, the interquartile range/median ratio (IQR/M) was kept below $30 \%$ and the reliability measurement index (RMI) values at 0.4 and above.

Table 2. Comparison of the mean SMV values across groups

\begin{tabular}{lccc}
\hline & Group & Stiffness value $(\mathrm{m} / \mathrm{s})$ & P-value \\
\hline SMG (right) & 1 & $2.22 \pm 1.52$ & 0.009 \\
& 2 & $1.93 \pm 1.08$ & \\
SMG (left) & 1 & $2.37 \pm 1.60$ & 0.006 \\
& 2 & $2.02 \pm 1.08$ & \\
PG (right) & 1 & $3.14 \pm 2.23$ & $<0.001$ \\
& 2 & $2.17 \pm 1.21$ & \\
PG (left) & 1 & $3.17 \pm 2.20$ & $<0.001$ \\
& 2 & $2.08 \pm 1.21$ & \\
\hline
\end{tabular}

Mean SMV values, stiffness values; group 1, patient group ( $n=49)$; group 2, control group ( $n=49$ ); SMG, submandibular gland; $P G$, parotid gland.

the submandibular gland was 0.652 and the $95 \%$ confidence interval (CI) was $0.575-0.730$. Using a cutoff value of $1.95 \mathrm{~m} / \mathrm{s}$, the

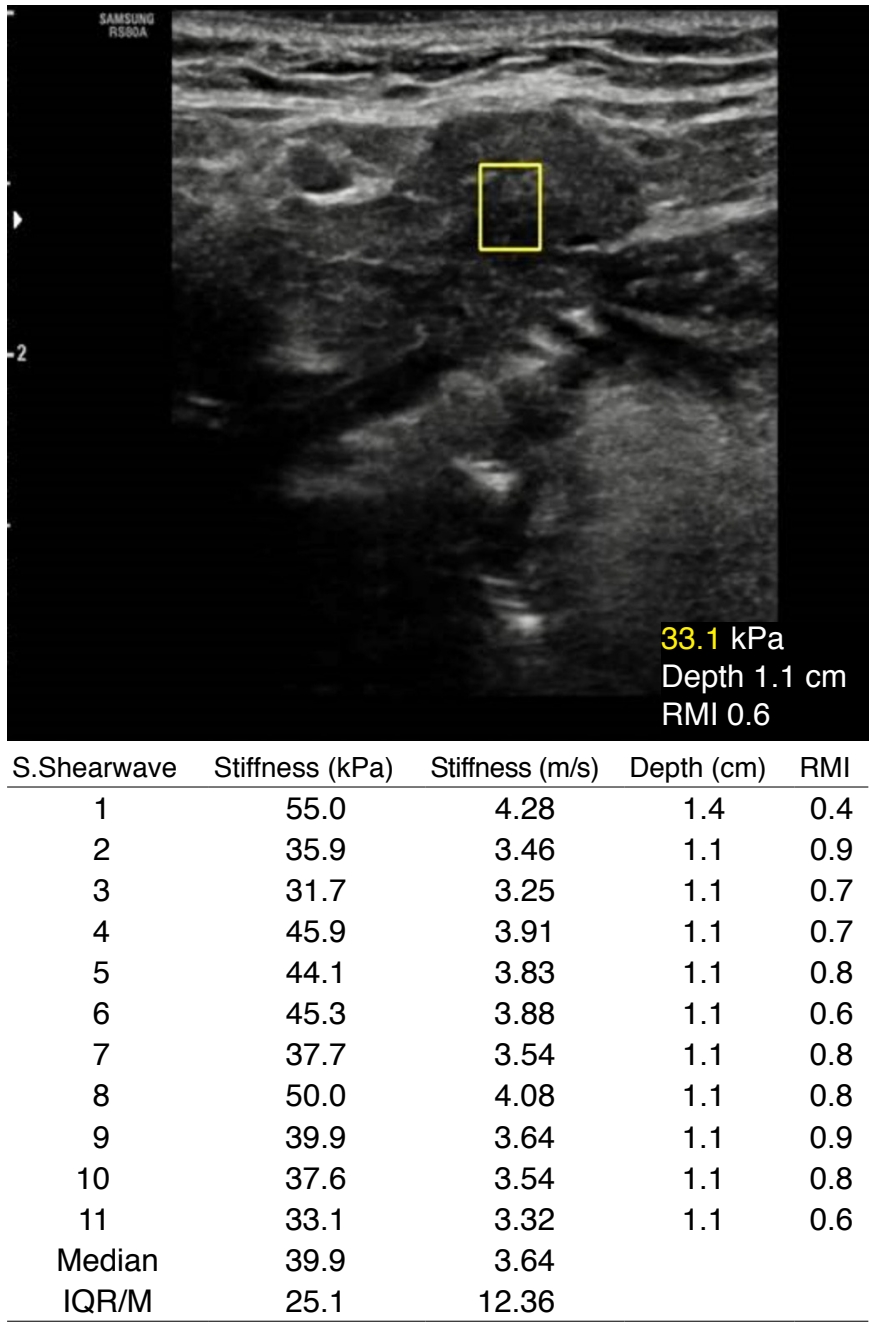

Fig. 5. Shear wave elastography (SWE) measurements in the submandibular gland. The figure presents SWE measurements of the submandibular gland. For each salivary gland measurement, the interquartile range/median ratio (IQR/M) was kept below 30\% and the reliability measurement index (RMI) values at 0.4 and above.

sensitivity, specificity, PPV, and NPV were $69.4 \%, 52 \%, 59.1 \%$, and $63 \%$, respectively. The AUROC for the parotid gland was 0.887 and the $95 \% \mathrm{Cl}$ was $0.839-0.936$. Using a cutoff value of $2.39 \mathrm{~m} / \mathrm{s}$, the sensitivity, specificity, PPV, and NPV were $82.7 \%, 83.7 \%, 83.5 \%$, and $83.8 \%$, respectively. The diagnostic performance of MSGUS and SWE for major salivary gland involvement in SjS is summarized in Table 3.

In SjS patients, the mean SWV values of the parotid gland were significantly higher than those of the submandibular gland (right side: $3.14 \pm 2.23 \mathrm{~m} / \mathrm{s}$ vs. $2.22 \pm 1.52 \mathrm{~m} / \mathrm{s}, P<0.05$; left side: $3.17 \pm 2.20$ $\mathrm{m} / \mathrm{s}$ vs. $2.37 \pm 1.60 \mathrm{~m} / \mathrm{s}, \mathrm{P}<0.05)$. In SjS patients, no statistically significant differences in elasticity were found between the rightside submandibular gland and parotid glands and the corresponding 


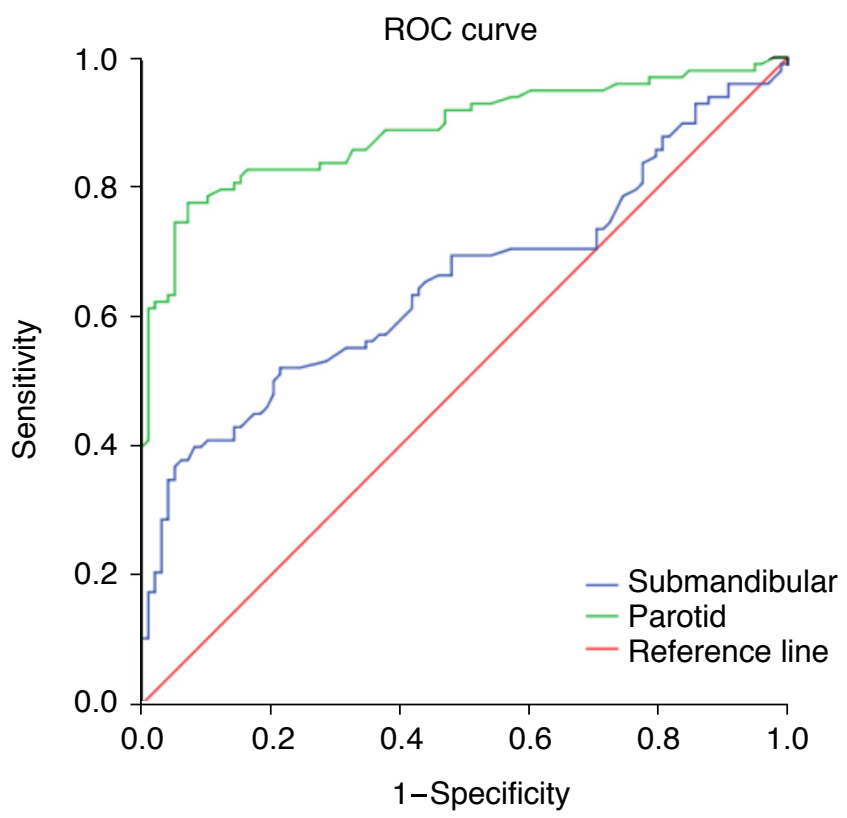

Fig. 6. Receiver operating characteristic (ROC) curve of shear wave velocity values for salivary gland involvement of Sjögren syndrome. The area under ROC curve and the cut-off value of shear wave velocity measurements were 0.652 and $1.95 \mathrm{~m} / \mathrm{s}$ for submandibular gland, and 0.887 and $2.39 \mathrm{~m} / \mathrm{s}$ for parotid gland, respectively.

Table 3. The diagnostic performance of MSGUS and SWE in major salivary gland involvement of SjS

\begin{tabular}{lcccc}
\hline Parameter & Sensitivity (\%) & Specificity (\%) & PPV (\%) & NPV (\%) \\
\hline MSGUS & & & & \\
SMG & 69.4 & 73.5 & 72.3 & 70.6 \\
PG & 69.4 & 65.3 & 66.7 & 68.1 \\
SWE & & & & \\
SMG & 69.4 & 52.0 & 59.1 & 63.0 \\
PG & 82.7 & 83.7 & 83.5 & 82.8 \\
\hline
\end{tabular}

MSGUS, major salivary gland ultrasonography; SWE, shear wave elastography; SjS, Sjogren syndrome; PPV, positive predictive value; NPV, negative predictive value; SMG, submandibular gland; PG, parotid gland.

left-side glands. Spearman correlation analysis showed a Spearman correlation analysis showed a high level of correlation between the each sides values of the submandibular glands $(P<0.001, r=0.745)$, and a moderate correlation between the each sides values of the parotid glands $(P<0.001, r=0.679)$. When the elasticity values of the bilateral salivary glands were compared according to the duration of the disease and focus scores in SjS patients, no statistically significant relationships were found ( $P>0.05)$.

The MSGUS and PSWE images, which were statistically significant for the diagnosis of salivary gland involvement in SjS patients, were evaluated together by logistic regression modeling. In model 1 , it was observed that sensitivity and specificity did not increase when SWE was added to MSGUS imaging for the evaluation of the submandibular gland (sensitivity, $69.4 \%$ vs. $69.4 \%$; specificity, $73.5 \%$ vs. $52 \%$ ). The OR for the submandibular gland was 0.375 ( $95 \% \mathrm{Cl}, 0.185$ to 0.690$)$ for pSWE and $0.148(95 \% \mathrm{Cl}, 0.078$ to 0.283 ) for MSGUS. In model 2 , it was observed that sensitivity and specificity increased when SWE was added to MSGUS imaging for the evaluation of the parotid gland (sensitivity, $69.4 \%$ vs. $82.7 \%$; specificity, $65.3 \%$ vs. $83.7 \%)$. The ORs for the parotid gland were $0.046(95 \% \mathrm{Cl}, 0.021$ to 0.099$)$ for pSWE and $0.296(95 \% \mathrm{Cl}, 0.136$ to 0.641$)$ for MSGUS.

\section{Discussion}

While objective tests with high accuracy are available to detect lacrimal gland involvement in SjS, there are no specific tests with sufficient diagnostic validity to show salivary gland involvement. The development of noninvasive, reliable, repeatable, and accurate diagnostic strategies with high diagnostic performance for salivary gland involvement would significantly benefit SjS patients. MSGUS is a noninvasive, easily accessible, inexpensive, radiation-free, and reproducible method used to evaluate the major salivary glands to aid in the diagnosis of SjS. There are many studies in the literature on the evaluation of major salivary gland involvement in SjS using gray-scale US. These studies have shown a wide variation in sensitivity $(46 \%-92 \%)$ and specificity $(73 \%-98 \%)$ of MSGUS for SjS $[2,3,7,9,23,24]$. The wide range of diagnostic performance may result from the use of different semi-quantitative and operatordependent scoring systems for parenchymal changes. In our study, a new grading system was made using a scoring system similar to that of previous studies $[2,3,7,24]$. The sensitivity and specificity of this new grading system were found to be $69.4 \%$ and $73.5 \%$ for the submandibular gland and $69.4 \%$ and $65.3 \%$ for the parotid gland, respectively.

SWE, which has been used in recent years, is a new and noninvasive imaging method that quantitatively evaluates tissue stiffness. Lymphocytic infiltration, hyperplasia of ductal epithelium cells, and fibrosis of salivary glands are conditions that cause increased parenchymal stiffness and higher SWV values in SjS patients [25]. There are a few published studies regarding the contribution of SWE imaging to the diagnosis of primary SjS. In these studies, the sensitivity and specificity ranged widely for the submandibular gland $(65 \%-87 \%$ and $44 \%-92 \%$, respectively) and for the parotid gland ( $59 \%-92 \%$ and $50 \%-91 \%$, respectively) $[17,18,26-28]$. The sensitivity and specificity rates of SWE were found to be $69.4 \%$ and $52 \%$ for the submandibular gland and 
$82.7 \%$ and $83.7 \%$ for the parotid gland, respectively, in our study. The optimal cutoff value for the parotid gland in our study $(2.39 \mathrm{~m} /$ s) was similar to the value of $2.395 \mathrm{~m} / \mathrm{s}$ reported by Knopf et al. [18] and close to the value of $2.34 \mathrm{~m} / \mathrm{s}$ reported by Hofauer et al. [26]. The optimal cutoff value for the submandibular glands in our study $(1.95 \mathrm{~m} / \mathrm{s})$ was similar to the value of $1.95 \mathrm{~m} / \mathrm{s}$ reported by Hofauer et al. [26]. The population in the study of Hofauer et al. [26] was similar to that of the present study; however, their study reported higher diagnostic performance rates than this study.

Differences in cutoff values and diagnostic performance between the present study and other studies may be due to differences in sample size and the selection of different control groups.

Unlike many previous studies, in the present study, the diagnostic performance of different ultrasonographic imaging modalities (pSWE and MSGUS) was examined separately and in combination. When PSWE and MSGUS were used together, there was a significant improvement in diagnostic performance for parotid gland involvement compared to evaluations using MSGUS only (sensitivity, $69.4 \%$ vs. $82.7 \%$; specificity, $65.3 \%$ vs. $83.7 \%$ ). The OR for the parotid gland was $0.046(95 \% \mathrm{Cl}, 0.021$ to 0.099$)$ in pSWE and $0.296(95 \% \mathrm{Cl}, 0.136$ to 0.641$)$ in MSGUS. In the literature, only a preliminary study by Chen et al. [28] investigated the diagnostic performance of combined MSGUS and SWE, and they found that the combination of MSGUS and SWE increased the sensitivity, but decreased the specificity in the evaluation of salivary glands. The principal difference of the present study from this preliminary study is the usage of a new grading system for MSGUS.

In the SjS patient group in the present study, the mean SWV values for the parotid glands were statistically significantly higher than the values of the submandibular glands, similar to previous reports in the literature (AUROC, 0.657-0.887; $P<0.001$ to $\mathrm{P}=0.009$ ). The serous parotid gland is primarily involved in $\mathrm{SjS}$, while the submandibular gland, with a mixed structure, is less affected. It has also been reported that lymphocytic infiltration, loss of acini, and the development of fibrosis may be more pronounced and/or may occur earlier in the parotid glands than in the submandibular glands [29]. In addition, Golder and Stiller [30], in their study analyzing the results of sialography; the lymphocytic infiltration observed in primary SjS patients was more severe in the parotid glands than in the submandibular glands. These histopathological changes and fibrosis, which may develop earlier and/or to a greater degree in parotid glands than in submandibular glands, may explain the significant difference in the mean stiffness values of parotid glands obtained in the present study. For this reason, the parotid gland should be the first choice of salivary gland in SWE imaging to evaluate salivary gland involvement in SjS.

This study has several limitations. First, this is a single-center study with a relatively limited sample size. Further studies with larger sample sizes are needed to confirm the findings of this study. The second limitation is that in some cases, the diagnosis was reached only by clinical, laboratory, and imaging methods without a histopathological diagnosis. In addition, the fact that most of the patients with SjS in our study were newly diagnosed and followed up in the early stage made it difficult to analyze the mean SWV values according to the duration of the disease. The third limitation is that intraobserver and interobserver agreement was not evaluated. While making measurements, care was taken to ensure that the RMI values and IQR/M were within the specified range. However, the lack of an evaluation of intraobserver and interobserver agreement means that the reliability of the SWV measurements was not sufficiently established.

There has been a great deal of interest and effort to include US of the major salivary glands in the SjS classification criteria. The diagnostic efficacy of MSGUS in detecting salivary gland involvement in patients with early-stage SjS is limited, but it is thought that the addition of SWE to the MSGUS examination and a combined evaluation, especially for the parotid gland, may increase the diagnostic effectiveness of US for early-stage SjS.

ORCID: Yunus Emre Oruk: https://orcid.org/0000-0003-1901-7825; Mehmet Burak Çildağ: https://orcid.org/0000-0003-2371-3540; Can Zafer Karaman: https://orcid.org/00000001-5462-321X; Songül Çildağ: https://orcid.org/0000-0001-9617-0925

\section{Author Contributions}

Conceptualization: Oruk YE, Çildağ MB, Karaman CZ. Data acquisition: Oruk $Y E$, Çildağ $S$. Data analysis or interpretation: Oruk YE. Drafting of the manuscript: Oruk YE, Çildağ MB. Critical revision of the manuscript: Oruk $Y E$, Çildağ $M B$, Karaman $C Z$, Çildağ $S$. Approval of the final version of the manuscript: all authors.

\section{Conflict of Interest}

No potential conflict of interest relevant to this article was reported.

\section{Acknowledgments}

The authors would like to thank Dilara Özen Oruk, PT and Veli Süha Öztürk, MD for assistance with the statistical analysis. The research project was supported by a grant from the Adnan Menderes University Research Projects Coordination Office (project grant number TPF18075, with the title "Effectiveness of Ultrasonography and Shear Wave Sonoelastography in Sjogren's Syndrome with Salivary Gland Involvement"). 


\section{References}

1. Ramos-Casals M, Font J. Primary Sjogren's syndrome: current and emergent aetiopathogenic concepts. Rheumatology (Oxford) 2005;44:1354-1367.

2. Hocevar A, Ambrozic A, Rozman B, Kveder T, Tomsic M. Ultrasonographic changes of major salivary glands in primary Sjogren's syndrome: diagnostic value of a novel scoring system. Rheumatology (Oxford) 2005;44:768-772.

3. Milic VD, Petrovic RR, Boricic IV, Radunovic GL, Pejnovic NN, Soldatovic I, et al. Major salivary gland sonography in Sjogren's syndrome: diagnostic value of a novel ultrasonography score (0-12) for parenchymal inhomogeneity. Scand J Rheumatol 2010;39:160166.

4. Tzioufas AG, Kapsogeorgou EK, Moutsopoulos HM. Pathogenesis of Sjogren's syndrome: what we know and what we should learn. J Autoimmun 2012;39:4-8.

5. Shiboski CH, Shiboski SC, Seror R, Criswell LA, Labetoulle M, Lietman TM, et al. 2016 American College of Rheumatology/ European League Against Rheumatism classification criteria for primary Sjogren's syndrome: a consensus and data-driven methodology involving three international patient cohorts. Arthritis Rheumatol 2017;69:35-45.

6. Baldini C, Luciano N, Tarantini G, Pascale R, Sernissi F, Mosca M, et al. Salivary gland ultrasonography: a highly specific tool for the early diagnosis of primary Sjogren's syndrome. Arthritis Res Ther 2015;17:146.

7. Milic V, Petrovic R, Boricic I, Radunovic G, Marinkovic-Eric J, Jeremic $P$, et al. Ultrasonography of major salivary glands could be an alternative tool to sialoscintigraphy in the American-European classification criteria for primary Sjogren's syndrome. Rheumatology (Oxford) 2012;51:1081-1085.

8. Niemela RK, Takalo R, Paakko E, Suramo I, Paivansalo M, Salo $T$, et al. Ultrasonography of salivary glands in primary Sjogren's syndrome: a comparison with magnetic resonance imaging and magnetic resonance sialography of parotid glands. Rheumatology (Oxford) 2004;43:875-879.

9. Jousse-Joulin S, Milic V, Jonsson MV, Plagou A, Theander E, Luciano $\mathrm{N}$, et al. Is salivary gland ultrasonography a useful tool in Sjogren's syndrome? A systematic review. Rheumatology (Oxford) 2016;55:789-800.

10. Cornec D, Jousse-Joulin S, Saraux A, Devauchelle-Pensec V. Salivary gland ultrasound to diagnose Sjogren's syndrome: a claim to standardize the procedure. Rheumatology (Oxford) 2015;54:199200.

11. Theander E, Mandl T. Primary Sjogren's syndrome: diagnostic and prognostic value of salivary gland ultrasonography using a simplified scoring system. Arthritis Care Res (Hoboken) 2014;66:1102-1107
12. Al-Qahtani M. Shear-wave and strain elastography: a comparative review on principles, basic techniques and applications. Curr Med Imaging 2016;12:269-278.

13. Bercoff J, Tanter M, Fink M. Supersonic shear imaging: a new technique for soft tissue elasticity mapping. IEEE Trans Ultrason Ferroelectr Freq Control 2004;51:396-409.

14. Bhatia KS, Lee YY, Yuen EH, Ahuja AT. Ultrasound elastography in the head and neck. Part I. Basic principles and practical aspects. Cancer Imaging 2013;13:253-259.

15. Cosgrove D, Piscaglia F, Bamber J, Bojunga J, Correas JM, Gilja OH, et al. EFSUMB guidelines and recommendations on the clinical use of ultrasound elastography. Part 2: Clinical applications. Ultraschall Med 2013;34:238-253.

16. Turnaoglu H, Kural Rahatli F, Pamukcu M, Haberal KM, Uslu N. Diagnostic value of acustic radiation force impulse imaging in the assessment of salivary gland involvement in primary Sjogren's sydrome. Med Ultrason 2018;20:313-318.

17. Chen S, Wang Y, Chen S, Wu Q, Chen S. Virtual Touch quantification of the salivary glands for diagnosis of primary Sjogren syndrome. J Ultrasound Med 2016;35:2607-2613.

18. Knopf A, Hofauer B, Thurmel K, Meier R, Stock K, Bas M, et al. Diagnostic utility of acoustic radiation force impulse (ARFI) imaging in primary Sjoegren's syndrome. Eur Radiol 2015;25:3027-3034.

19. Sporea I, Gradinaru-Tascau O, Bota S, Popescu A, Sirli R, Jurchis $A$, et al. How many measurements are needed for liver stiffness assessment by 2D-shear wave elastography (2D-SWE) and which value should be used: the mean or median? Med Ultrason 2013;15:268-272.

20. Mulabecirovic A, Mjelle AB, Gilja OH, Vesterhus M, Havre RF. Repeatability of shear wave elastography in liver fibrosis phantoms Evaluation of five different systems. PLoS One 2018;13:e0189671.

21. Fang C, Jaffer OS, Yusuf GT, Konstantatou E, Quinlan DJ, Agarwal K, et al. Reducing the number of measurements in liver point shearwave elastography: factors that influence the number and reliability of measurements in assessment of liver fibrosis in clinical practice. Radiology 2018;287:844-852.

22. Choi K, Kong D, Hah Z, Lee HK. A reliability index of shear wave speed measurement for shear wave elastography. In: 2015 IEEE International Ultrasonics Symposium (IUS); 2015 Oct 21-24; Taipei, Taiwan. New York: Institute of Electrical and Electronics Engineers, 2015.

23. Delli K, Dijkstra PU, Stel AJ, Bootsma H, Vissink A, Spijkervet FK. Diagnostic properties of ultrasound of major salivary glands in Sjogren's syndrome: a meta-analysis. Oral Dis 2015;21:792-800.

24. Milic VD, Petrovic RR, Boricic IV, Marinkovic-Eric J, Radunovic $G L$, Jeremic PD, et al. Diagnostic value of salivary gland ultrasonographic scoring system in primary Sjogren's syndrome: a comparison with scintigraphy and biopsy. J Rheumatol 2009;36:1495-1500. 
25. Dewall RJ. Ultrasound elastography: principles, techniques, and clinical applications. Crit Rev Biomed Eng 2013;41:1-19.

26. Hofauer B, Mansour N, Heiser C, Gahleitner C, Thuermel K, Bas M, et al. Sonoelastographic modalities in the evaluation of salivary gland characteristics in Sjogren's syndrome. Ultrasound Med Biol 2016;42:2130-2139.

27. Samier-Guerin A, Saraux A, Gestin S, Cornec D, Marhadour T, Devauchelle-Pensec $V$, et al. Can ARFI elastometry of the salivary glands contribute to the diagnosis of Sjogren's syndrome? Joint
Bone Spine 2016;83:301-306.

28. Chen $S$, Wang Y, Zhang $G$, Chen $S$. Combination of salivary gland ultrasonography and virtual touch quantification for diagnosis of Sjogren's syndrome: a preliminary study. Biomed Res Int 2016;2016:2793898.

29. Dankof A, Morawietz L, Feist E. Labial salivary gland biopsy in Sjogren's syndrome. Pathologe 2006;27:416-421.

30. Golder W, Stiller M. Distribution pattern of Sjogren's syndrome: a sialographical study. Z Rheumatol 2014;73:928-933. 